Probl. Anal. Issues Anal. Vol. 5 (23), No. 1, 2016, pp. 31-44

DOI: $10.15393 /$ j3.art.2016.3370

UDC 511

B. M. Shirokov, L. A. Gromakovskaya

\title{
DISTRIBUTION OF VALUES OF THE SUM OF UNITARY DIVISORS IN RESIDUE CLASSES
}

\begin{abstract}
In this paper we prove the tauberian type theorem containing the asymptotic series for the Dirichlet series. We use this result to study distribution of sum of unitary divisors in residue classes coprime with a module. The divisor $d$ of the integer $n$ is an unitary divisor if $\left(d, \frac{n}{d}\right)=1$. The sum of unitary divisors of a number $n$ is denoted by $\sigma^{*}(n)$. For a fixed function $f(n)$ let us denote by $S(x, r)$ the numbers of positive integers $n \leq x$ such that $f(n) \equiv r(\bmod N)$ for $x>0$ and $r$ coprime with module $N$. According to W. Narkiewicz [5], a function $f(n)$ is called weakly uniformly distributed modulo $N$ if and only if for every pair of coprime integer $a, b$
\end{abstract}

$$
\lim _{x \rightarrow \infty} \frac{S(x, a)}{S(x, b)}=1
$$

provided that the set $\{n \mid(f(n), N)=1\}$ is infinite. We use the tauberian theorem to obtain an asymptotic series for $S(x, r)$ for $\sigma^{*}(n)$. Then we derive necessary and sufficient conditions for the module $N$ that provide weakly uniform distribution modulo $N$ of the function $\sigma^{*}(n)$.

Key words: sum of the unitary divisors, tauberian theorem, distribution of values in the residue classes

\section{Mathematical Subject Classification: 11 N69}

1. Introduction. Problems connected with distribution of values of arithmetical functions in residue classes have been considered from the middle of the XX century. Originally the purpose of these papers was to obtain asymptotics of the value set connected by the some congruent condition, mainly on a prime module (see, for example, [1, 2]). 
Paper by I. Niven [3] contains the concept of uniform distribution in residue classes and in ring $\mathbb{Z}$ and started studying conditions of uniform distribution in residue classes. S. Uchiyama [4] obtained necessary and sufficient conditions for uniform distribution of sequence on given module and established duality of this concept to the concept of uniform distribution of a sequence modulo 1 .

In paper [5] W. Narkiewicz transfered this concept to the residue classes coprime with a module. He called uniformly distribution in these classes weakly uniform and deduced test for weakly uniform distribution for some class of multiplicative functions. Using this test J. Śliva [6] obtained necessary and sufficient conditions on module that sum of divisors is weekly uniformly distributed on this module.

Let $N$ be some fixed integer. For some fixed function $f(n)$ let us denote by $S(x, r)$ the natural number $n \leq x$ for which $f(n) \equiv r(\bmod N)$ for $x>0$ and for $r$ coprime with some module $N$.

According to W. Narkiewicz [5] a function $f(n)$ is called weakly uniformly distributed modulo $N$ if for every pair of integer $a, b$ coprime with $N$

$$
\lim _{x \rightarrow \infty} \frac{S(x, a)}{S(x, b)}=1
$$

provided that the set $\{n \mid(f(n), N)=1\}$ is infinite.

The results of W. Narkiewicz [5] and J. Śliva [6] are transfered to a more wide class of multiplicative functions in [7]- [9].

Let us note the paper of O. M. Fomenko [10] that contains results on distribution of values in residue classes for functions which do not belong to Narkiewicz classes.

The asymptotic formulae for $S(x, r)$ in the papers contain only the main member of asymptotics of weekly uniform distribution of multiplicative functions. These results can be sharpen in most cases; this was done in [7] - 9]. The point is that usually the Delange tauberian theorem [11] is applied to generating the Dirichlet series. But generating functions used by this theorem have better properties than the theorem demands.

We prove the tauberian type theorem 1 in this paper, which can replace the Delange theorem to obtain sharper results.

This paper is devoted to study the weekly uniform distribution of values of sum of unitary divisors denoted by $\sigma^{*}(n)$.

Definition 1. A divisor $d$ of $n$ is called unitary divisor if $\left(n, \frac{n}{d}\right)=1$. 
Upper bound set of values of the function $\sigma^{*}(n)$ was studied in [12.

Let us introduce necessary notation. Let $N, a, b, d, i, j, k, l, m, n$ and $r$ be nonnegative integers; $p, q$ be prime numbers; $\chi, X$ be the Dirichlet characters and $\chi_{0}, X_{0}$ be the relevant principal characters; $(m, n)$ be the greatest common divisor of integers $m$ and $n$; $G(N)$ be the residue multiplicative group modulo $N$. We use notation $S(x, r)$ for the function $\sigma^{*}(n)$ in the sequel.

On the complex $s$-plane, $s=\sigma+i t$, for the some constants $c_{0}>0$ we denote $\sigma(t)=1-\frac{c_{0}}{\ln (2+|t|)},-\infty<t<\infty$ and

$$
\Omega=\left\{s=\sigma+i t \mid \sigma>\max \left\{\sigma(t), \frac{3}{4}\right\},-\infty<t<+\infty\right\}
$$

$\omega(n)$ is the number of distinct prime divisors of $n$;

$$
\lambda=\prod_{q \mid N} \frac{q-2}{q-1}, \quad \mu=(-1)^{\omega(N)} \prod_{q \mid N} \frac{1}{q-1} ;
$$

if $N=q_{1}^{k_{1}} \cdots q_{m}^{k_{m}}$ then let us denote $\bar{N}=q_{1} \cdots q_{m}$; let $\varphi(n)$ be Euler's function.

In this paper we prove the following theorems.

Theorem 1. Let for a complex-valued function $f(n)$ a number $A>0$ exist, such that $f(n)=O\left(\ln ^{A} n\right)$ and function $F(s)$ defined for $\sigma>1$ as sum of the series

$$
F(s)=\sum_{n=1}^{\infty} \frac{f(n)}{n^{s}}
$$

satisfy the following conditions:

1) there are a complex number $z$ and analytical in $\Omega$ function $G(s)$ such that $G(1) \neq 0$ and $F(s)$ can be extended to the region $\Omega$ by the equality

$$
F(s)=\frac{G(s)}{(s-1)^{z}}, \quad s \in \Omega ;
$$

2) there is a constant $c_{1}>0$ such that for the extension $F(s)$ the estimate

$$
F(s)=O\left(\ln ^{c_{1}}(2+|t|), \quad|t| \geq 1,\right.
$$

is correct.

Then there is a number $c>0$ such that 
a) if $z=0,-1,-2, \ldots$ then

$$
\sum_{n \leq x} f(n)=O\left(x e^{-c \sqrt{\ln x}}\right)
$$

b) if $z=1$ then

$$
\sum_{n \leq x} f(n)=G(1) x+O\left(x e^{-c \sqrt{\ln x}}\right)
$$

c) if $z$ is not integer then for any positive integer $n$

$$
\sum_{n \leq x} f(n)=\frac{x}{(\ln x)^{1-z}} P_{n-1}\left(\frac{1}{\ln x}\right)+O\left(\frac{x}{(\ln x)^{n+1-\operatorname{Re} z}}\right),
$$

where

$$
P_{n}(y)=\sum_{k=1}^{n} a_{k} \frac{(z-1) \cdots(z-k)}{\Gamma(z)} y^{k},
$$

$\Gamma(z)$ is the Euler gamma-function, $a_{k}$ are Taylor coefficients of the function $\frac{G(s)}{s}$ in point $s=1$.

Theorem 2. If $N$ is an odd number then for any positive integer $n$ and for any character $\chi$ modulo $N$ there are polynomials $P_{n}(y)$ and $Q_{n}(y, \chi)$ such that for any $r \in G(N)$ for $x \rightarrow \infty$

$$
\begin{gathered}
S(x, r)=\frac{x}{\varphi(N)}\left[\frac{1}{(\ln x)^{1-\lambda}}\left(P_{n}\left(\frac{1}{\ln x}\right)+O\left(\frac{1}{(\ln x)^{n+1}}\right)\right)+\right. \\
\left.+\sum_{\substack{\chi \neq \chi_{0} \\
\chi^{\varphi(N)}=\chi_{0}}} \frac{1}{(\ln x)^{1-\mu}}\left(Q_{n}\left(\frac{1}{\ln x}, \chi\right)+O\left(\frac{1}{(\ln x)^{n+1}}\right)\right)\right] .
\end{gathered}
$$

Summation is taken over the nonprincipal characters which have order $\varphi(\bar{N})$ in the character group modulo $N$ in last sum.

Theorem 3. The function $\sigma^{*}(n)$ is weakly uniformly distributed modulo $N$ if and only if $N$ is odd.

2. Proof of Theorem 1. We apply the approximate Perron formula with the half-integer $x$ for the sum of $f(n)$ values (see [13, p. 427]). Let 
$\sigma_{0}=1+\frac{2}{\ln x}$ and $T=e^{\sqrt{\ln x}}$. Let us choose any $c \in(0,1)$. Then $\ln ^{A} x \cdot e^{-\sqrt{\ln x}}=O\left(e^{-c \sqrt{\ln x}}\right)$. The Perron formula implies

$$
\sum_{n \leq x} f(n)=\frac{1}{2 \pi i} \int_{\sigma_{0}-i T}^{\sigma_{0}+i T} F(s) \frac{x^{s}}{s} d s+O\left(x e^{-c \sqrt{\ln x}}\right) .
$$

Let us apply the lemma from paper [7, p. 180] (see also [14, p. 14]) to the right-hand side integral. This lemma states that provided that $F(s)$ obeys assumptions of the theorem asymptotic formulae listed in the statement of the theorem hold for the integral. This proves Theorem 1.

3. Proof of Theorem 2. Let $N=q_{1}^{k_{1}} \cdots q_{m}^{k_{m}}$ and $q_{i}>2$. Using properties of characters we can present $S(x, r), r \in G(N)$, in the following form:

$$
S(x, r)=\sum_{\substack{n \leq x \\ \sigma^{*}(n) \equiv r(\bmod N)}} 1=\frac{1}{\varphi(N)} \sum_{\chi} \bar{\chi}(r) \sum_{n \leq x} \chi\left(\sigma^{*}(n)\right) .
$$

Let us denote

$$
S(x, \chi)=\sum_{n \leq x} \chi\left(\sigma^{*}(n)\right) .
$$

Then $S(x, r)$ takes the form

$$
S(x, r)=\frac{1}{\varphi(N)} S\left(x, \chi_{0}\right)+\frac{1}{\varphi(N)} \sum_{\chi \neq \chi_{0}} \bar{\chi}(r) S(x, \chi) .
$$

The sum $S(x, \chi)$ is sum of coefficients of the Dirichlet series:

$$
F(s . \chi)=\sum_{n=1}^{\infty} \frac{\chi\left(\sigma^{*}(n)\right)}{n^{s}} .
$$

Thus the problem is reduced to applying Theorem 1 to the Dirichlet series (8). To do this we need to study analytic properties of the function $F(s, \chi)$ in the domain $\Omega$.

The function $F(s, \chi)$ is analytic in the half-plane $\sigma>1$ as $|\chi(n)| \leq 1$. Since the series (8) converges absolutely for $\sigma>1$ but the function $\sigma^{*}(n)$ is multiplicative and $\sigma^{*}\left(p^{k}\right)=p^{k}+1$, the Euler theorem implies

$$
F(s, \chi)=\prod_{p}\left(1+\frac{\chi(p+1)}{p^{s}}+\frac{\chi\left(p^{2}+1\right)}{p^{2 s}}+\ldots\right) .
$$


Let us denote

$$
\begin{gathered}
A_{1}(s, \chi)=\left(1-\frac{\chi(3)}{2^{s}}\right)\left(1+\sum_{k=1}^{\infty} \frac{\chi\left(2^{k}+1\right)}{2^{k s}}\right) \prod_{p \mid N}\left(1+\sum_{k=1}^{\infty} \frac{\chi\left(p^{k}+1\right)}{p^{k s}}\right), \\
A_{2}(s, \chi)=\prod_{\substack{p \nmid N \\
p>2}}\left(1+\sum_{k=2}^{\infty} \frac{\chi\left(p^{k}+1\right)-\chi(p+1) \chi\left(p^{k-1}+1\right)}{p^{k s}}\right), \\
H(s, \chi)=\prod_{p \nmid N}\left(1-\frac{\chi(p+1)}{p^{s}}\right)^{-1}
\end{gathered}
$$

and assume that $A(s, \chi)=A_{1}(s, \chi) \cdot A_{2}(s, \chi)$. Then for $\sigma>1$ the function $F(s, \chi)$ takes the form

$$
F(s, \chi)=H(s, \chi) \cdot A(s, \chi) .
$$

This representation allows extension of $F(s, \chi)$ to the domain $\Omega$.

Consider the function $A(s, \chi)$. The factor

$$
\left(1-\frac{\chi(3)}{2^{s}}\right) \prod_{p \mid N}\left(1+\sum_{k=1}^{\infty} \frac{\chi\left(p^{k}+1\right)}{p^{k s}}\right)
$$

is the function that is regular, bounded, and does not vanish for $\sigma \geq 3 / 4$. Product taken over $p \mid N$ does not vanish in the same half-plane. The sum of the series

$$
1+\sum_{k=1}^{\infty} \frac{\chi\left(2^{k}+1\right)}{2^{k s}}
$$

can vanish for $s=1$ if and only if $\chi\left(2^{k}+1\right)=-1$ for any natural $k$. But it is impossible because if $\chi(3)=-1$ then $\chi\left(2^{3}+1\right)=\chi(9)=\chi^{2}(3)=1$.

For sum of the series in the product $A_{2}(s, \chi)$ for $\sigma \geq 3 / 4$ the estimation

$$
\left|\sum_{k=2}^{\infty} \frac{\chi\left(p^{k}+1\right)-\chi(p+1) \chi\left(p^{k-1}+1\right)}{p^{k s}}\right| \leq \frac{2}{p^{\sigma}\left(p^{\sigma}-1\right)} \leq \frac{2}{3^{\sigma}\left(3^{\sigma}-1\right)} .
$$

is correct. The number in the right-hand side is less that 1 since $3^{3 / 4}>$ $>2$. Thus each member of the product $A_{2}(s, \chi)$ does not vanish and the product converges absolutely for $\sigma \geq 3 / 4$. Therefore the function $A_{2}(s, \chi)$ is regular, bounded in region $\Omega$ and does not vanish for $s=1$. 
Let us consider $\ln H(s, \chi)$ and note that the series

$$
\sum_{p \nmid N} \ln \left(1-\frac{\chi(p+1)}{p^{s}}\right)
$$

converges absolutely for $\sigma>1$ because the series composed from the real and imaginary parts of this series converge absolutely; all logarithm branches are important here. Therefore for some branch we have

$$
\ln \prod_{p \nmid N}\left(1-\frac{\chi(p+1)}{p^{s}}\right)^{-1}=-\sum_{p \nmid N} \ln \left(1-\frac{\chi(p+1)}{p^{s}}\right) .
$$

Therefore

$$
\ln H(s, \chi)=\sum_{p} \frac{\chi(p+1)}{p^{s}}+\sum_{p} \sum_{k \geq 2} \frac{\chi^{k}(p+1)}{p^{k s}} .
$$

Let us denote by $B(s, \chi)$ the sum of the series for $k \geq 2$. This function is regular and bounded for $\sigma \geq 3 / 4$. In fact, the estimate

$$
\left|\sum_{k \leq 2} \frac{\chi^{k}(p+1)}{p^{k s}}\right| \leq \sum_{k \geq 2} \frac{1}{p^{\sigma}\left(p^{\sigma}-1\right)}=O\left(p^{-3 / 2}\right)
$$

implies that the series converges absolutely and uniformly in the described domain.

The first sum taken over prime numbers in equality (9) contains necessary information. Let us transform it using the character properties:

$$
\sum_{p} \frac{\chi(p+1)}{p^{s}}=\sum_{r \in G(N)} \sum_{p \equiv r(\bmod N)} \frac{\chi(p+1)}{p^{s}}=\sum_{r \in G(N)} \sum_{p} \delta(p, r) \frac{\chi(p+1)}{p^{s}},
$$

where $\delta(p, r)$ is equal to 1 if $p(\bmod N)=r$ and equal to 0 otherwise. The orthogonality property of characters $X(n)$ is the following formula:

$$
\delta(p, r)=\frac{1}{\varphi(N)} \sum_{X} \bar{X}(r) X(p) .
$$

Therefore

$$
\sum_{p} \frac{\chi(p+1)}{p^{s}}=\sum_{X} \frac{1}{\varphi(N)} \sum_{r \in G(N)} \chi(r+1) \bar{X}(r) \sum_{p} \frac{X(p)}{p^{s}} .
$$


Let us denote

$$
z(\chi, X)=\frac{1}{\varphi(N)} \sum_{r \in G(N)} \chi(r+1) \bar{X}(r) .
$$

The sum in the right-hand side of $(10)$ is over prime numbers and contains all necessary information about the Dirichlet $L$-function $L(S, X)$ :

$$
\sum_{p} \frac{X(p)}{p^{s}}=\ln L(s, X)-\sum_{p} \sum_{k \geq 2} \frac{X\left(p^{k}\right)}{p^{k s}} .
$$

The logarithm branch is selected in the same way as on the page 37 .

Let

$$
C(s, X)=-\sum_{p} \sum_{k \geq 2} \frac{X\left(p^{k}\right)}{p^{s}} .
$$

This function is also regular and bounded for $\sigma \geq 3 / 4$ : this is shown in the same way as for $B(s, \chi)$. Equalities 10 -13) allow representing $F(s, \chi)$ in the following form:

$$
F(s, \chi)=A(s, \chi) e^{B(s, \chi)} \prod_{X} e^{z(\chi, X) C(s, X)} \cdot[L(s, X)]^{z(\chi, X)} .
$$

It is well known that functions $L(s, X)$ for every $X \neq X_{0}$ and $(s-1) L\left(s, X_{0}\right)$ are regular in $\Omega$ and do not vanish for $s=1$ (see for example [13, ch. IV]). Let us denote

$$
z(\chi)=z\left(\chi, X_{0}\right)
$$

Thus the function

$$
\begin{aligned}
G(s, \chi) \stackrel{\text { def }}{=} A(s, \chi) e^{B(s, \chi)} \prod_{X} e^{z(\chi, X) C(s, X)} \cdot \prod_{X \neq X_{0}}[L(s, X)]^{z(\chi, X)} \times \\
\times\left[(s-1) L\left(s, X_{0}\right)\right]^{z(\chi)}
\end{aligned}
$$

is regular in $\Omega$ and $G(1, \chi) \neq 0$. Thus equalities 14 and 16 imply

$$
F(s, \chi)=\frac{G(s, \chi)}{(s-1)^{z(\chi)}} .
$$


Last equality allows extension of the function $F(s, \chi)$ to $\Omega$ so that $F(s, \chi)$ satisfies condition (1) of Theorem 1 with exponent $z=z(\chi)$.

It is known that $L(s, \chi)=O(\ln (2+|t|)$ in $\Omega$ for $t \rightarrow \infty$ (see [13, p. 132]). Equality (14) and properties of functions $A(s, \chi), B(s, \chi)$ and $C(s, \chi)$ imply that there is a constant $c_{1}>0$ such that

$$
F(s, \chi)=O\left(\ln ^{c_{1}}(2+|t|)\right)
$$

in $\Omega$ for $|t| \rightarrow \infty$.

This estimate shows that function $F(s, \chi)$ satisfies the condition (2). The last step to make is to calculate the exponent $z(\chi)$ for each character modulo $N$.

Let $N_{i}=q_{i}^{k_{i}}$. Then $N=N_{1} \cdots N_{m}$ and $G(N)=G\left(N_{1}\right) \times \cdots \times G\left(N_{m}\right)$. The character $\chi(n)$ modulo $N$ is defined by equality

$$
\chi(n)=\chi_{1}(n) \cdots \chi_{m}(n),
$$

where $\chi_{i}$ is the character modulo $N_{i}$. Since the Euler function is multiplicative equality 15 implies

$$
z(\chi)=\prod_{i=1}^{m} \frac{1}{\varphi\left(N_{i}\right)} \sum_{r \in G\left(N_{i}\right)} \chi_{i}(r+1)=\prod_{i=1}^{m} z\left(\chi_{i}\right) .
$$

Let us calculate the exponent $z(\chi)$ for the character $\chi(n)$ modulo $N=$ $=q^{k}$. There are two possibilities, one of them is divided in two subcases.

1. $\chi=\chi_{0}$.

Every $r \in G(N)$ is represented uniquely in the form

$$
r=a q+b, \quad 0 \leq a \leq q^{k-1}-1, \quad 1 \leq b \leq q-1 .
$$

Consider the transformation $r \rightarrow r+1$. Each residue $a q+q-1$ turns to residue $a q+q$ on which the character vanishes. Besides, residues $a q+1$ for each $a$ disappear after such transformation. Thus the number of residues $r+1$ for which $\chi_{0}=1$ equals $q^{k-1}(q-2)$. Therefore

$$
z\left(\chi_{0}\right)=\frac{q^{k-1}(q-2)}{\varphi(N)}=\frac{q-2}{q-1} .
$$


Using characters properties, in the same way as above, we conclude that

$$
\sum_{r \in G(N)} \chi(r+1)=\sum_{r \in G(N)} \chi(r)-\sum_{a=0}^{q^{k-1}-1} \chi(a q+1)=-\sum_{a=0}^{q^{k-1}-1} \chi(a q+1) .
$$

Let us calculate the last sum. Let $g$ be the primitive root modulo $q^{k}$ and $l=\operatorname{ind}_{g} r$ for $r=a q+1$. This means that $r \equiv g^{l} \equiv 1(\bmod q)$. Then $l \equiv 0(\bmod (q-1))$. Therefore an integer $n, 0 \leq n \leq q^{q-1}-1$ corresponds to each coefficient $a$ one-to-one so that

$$
a q+1 \equiv g^{n(q-1)}\left(\bmod q^{k}\right) .
$$

The cyclic group character $\chi$ is defined uniquely by the value $\chi(g)$ as one of the values of $\sqrt[N]{1}$. Therefore the equality (22) implies

$$
\chi(a q+1)=(\chi(g))^{n(q-1)} .
$$

For the brevity of notation let us assume $\zeta=(\chi(g))^{n(q-1)}$. Then

$$
z(\chi)=-\frac{1}{\varphi(N)} \sum_{n=0}^{q^{k-1}-1} \zeta^{n} .
$$

a) $\zeta=1$.

This means that the character $\chi$ has order $q-1$ in the character group modulo $N$. Then

$$
z(\chi)=-\frac{q^{k-1}}{\varphi\left(q^{k}\right)}=-\frac{1}{q-1} .
$$

b) $\zeta \neq 1$.

In this case

$$
z(\chi)=-\frac{1-\zeta^{q^{k-1}}}{\varphi(N)(1-\zeta)}=0,
$$

since $\zeta^{q^{k-1}}=(\chi(g))^{\varphi(N)}=1$.

Now on the base of the formula (19) for $N=N_{1} \cdots N_{m}$ we obtain three possible forms of the exponent $z(\chi)$ : 
1) $\chi=\chi_{0}$. In this case

$$
z\left(\chi_{0}\right)=\prod_{q \mid N} \frac{q-2}{q-1}=\lambda
$$

2) $\chi \neq \chi_{0}$, but $\chi^{\varphi(\bar{N})}=\chi_{0}$. Then

$$
z(\chi)=(-1)^{\omega(N)} \prod_{q \mid N} \frac{1}{q-1}=\mu .
$$

3) There is a factor $\chi_{i}$ in the produc $\chi=\chi_{1} \cdots \chi_{m}$ that $\chi_{i}^{q-1} \neq\left(\chi_{i}\right)_{0}$, i.e., $\chi^{\varphi(\bar{N})} \neq \chi_{0}$. Then $z(\chi)=0$.

In the first case $z\left(\chi_{0}\right)=\lambda$ is not integer. Then Theorem 1 implies that for every positive integer $n$ there is a polynomial $P_{n}(y)$ of power $n$ such that

$$
S\left(x, \chi_{0}\right)=\frac{x}{(\ln x)^{1-\lambda}}\left(P_{n}\left(\frac{1}{\ln x}\right)+O\left(\frac{1}{(\ln x)^{n+1}}\right)\right) .
$$

In the second case, i.e., a character $\chi \neq \chi_{0}$ exists and has order $\varphi(\bar{N})$ but $z(\chi)=\mu$ is not integer, Theorem 1 also implies existence of a polynomial $Q(y, \chi)$ of power $n$ for all positive integer $n$ such that

$$
S(x, \chi)=\frac{x}{(\ln x)^{1-\mu}}\left(Q_{n}\left(\frac{1}{\ln x}, \chi\right)+O\left(\frac{1}{(\ln x)^{n+1}}\right)\right) .
$$

In the third case, when $z(\chi)=0$, Theorem 1 implies

$$
S(x, \chi)=O\left(x e^{-c \sqrt{\ln x}}\right) .
$$

Now substituting $S(x, \chi)$ for all forms of $z(\chi)$ to formula (7), we obtain asymtotic equality of Theorem 2. This finishes the proof of Theorem 2.

4. Proof of Theorem 3. The proof is based on the following test by W. Narkiewicz [5].

A function $f(n)$ is weekly uniformly distributed $(\bmod N)$ if and only if for every nonprincipal character $\chi(\bmod N)$ the following evaluation holds

$$
\sum_{n \leq x} \chi(f(n))=o\left(\sum_{n \leq x} \chi_{0}(f(n))\right.
$$


for $x \rightarrow \infty$.

If $N$ is odd then formulae (26)-28) imply equality (30). Therefore if $N$ is odd then $\sigma^{*}(n)$ is weekly uniformly distributed.

Let $N$ be even. If $p^{\alpha} \mid N, p>2, \alpha \geq 1$ then

$$
\sum_{n \leq x} \chi(f(n))=o\left(\sum_{n \leq x} \chi_{0}(f(n))\right.
$$

but $p^{\alpha}+1$ is even. Therefore

$$
\chi\left(\sigma^{*}(n)\right)=0
$$

for every character modulo N. Therefore

$$
S(x, \chi)=\sum_{n \leq x} \chi\left(\sigma^{*}(n)\right)=\sum_{2^{k} \leq x} \chi\left(2^{k}+1\right)
$$

Let $N=2^{m}, m \geq 2$. For $k \geq m$ and for every character $\chi(\bmod N)$ we have $\chi\left(2^{k}+1\right)=1$, since the congruence $2^{k}+1 \equiv 1(\bmod N)$ holds. Therefore for every character $\chi(\bmod N)$

$$
S(x, \chi)=\sum_{k=1}^{m-1} \chi\left(2^{k}+1\right)+\sum_{m \leq k \leq \frac{\ln x}{\ln 2}} 1=\frac{\ln x}{\ln 2}+O(1)
$$

Thus equality 30 is wrong.

Let now $N=2^{m} Q, \quad m \geq 1$ and $Q$ be odd. If for every $\chi \neq \chi_{0}$ equality (30) holds then

$$
\sum_{\chi \neq \chi_{0}} S(x, \chi)=o\left(S\left(x, \chi_{0}\right)\right)
$$

Therefore

$$
\sum_{\chi} S(x, \chi) \sim S\left(x, \chi_{0}\right)
$$

But it is wrong. In fact,

$$
\sum_{\chi} S(x, \chi)=\sum_{k \leq \frac{\ln x}{\ln 2}} \sum_{\chi} \chi\left(2^{k}+1\right) .
$$


If $\left(2^{k}+1, Q\right)>1$ then the last sum vanishes. Therefore the sum for $k \leq \frac{\ln x}{\ln 2}$ in equality 32 contains only members for which $\left(2^{k}+1, N\right)=1$. But if $(n, N)=1$ then

$$
\sum_{\chi} \chi(n)= \begin{cases}\varphi(N), & n \equiv 1(\bmod N) \\ 0, & n \neq 1(\bmod N) .\end{cases}
$$

But the congruence $2^{k}+1 \equiv 1(\bmod N)$ implies the congruence $2^{k}+1 \equiv$ $\equiv 1(\bmod Q)$, that is $2^{k} \equiv 0(\bmod Q)$; this is impossible for any natural $k$. Therefore

$$
\sum_{\chi} S(x, \chi)=0
$$

Formula (31) implies equality $S\left(x, \chi_{0}\right)=0$ since the values of this sum are integer. This equality implies $\chi_{0}\left(2^{k}+1\right)=0$ for every natural $k$ since the values of the principal character are 0 and 1 . But it means that for every $k$

$$
d_{k}=\left(2^{k}+1, Q\right)>1
$$

But for every integer $l$

$$
2^{l \varphi(Q)}+1 \equiv 2(\bmod Q) .
$$

for $k=l \varphi(Q)$. This congruence holds for module $d_{k}$ since the number $Q$ is divided by $d_{k}$ for every $k$. In other words, $d_{k}$ is coprime with the number $2^{k}+1$ but this contradicts to inequality (33). This contradiction proves that relation (31) is wrong. Therefore the function is not weekly uniformly distributed. Theorem 3 is proved.

\section{References}

[1] Sathe L. G. On a congruence property of the divisor functions. Amer. J. Math., vol. 67, pp. 397-406.

[2] Rankin R. A. The distribution of divisor functions. Proc. Glasgow Math. Assoc., 1961, vol. 5, no. 1, pp. 35-40.

[3] Niven I. Uniform distribution of sequences of integers. Trans. Amer. Math. Soc., 1961, vol. 98, pp. 52-61.

[4] Uchiyama S. On the uniform distribution of siquences of integer. Proc. Japan Acad., 1961, vol. 37, pp. 605-609. 
[5] Narkievicz W. On distribution of values of multiplicative functions in residue classes. Acta Arithm., 1967, vol. 12, no. 3, pp. 269-279.

[6] Śliva J. On distribution of values of $\sigma(n)$ in residue classes. Colloq. Math., 1973, vol. 27, no. 2, pp. 283-271.

[7] Shirokov B. M. A distribution of arithmetic functions in residue classes. Zapiski nauch. sem., 1983, vol. 121, pp. 176-186.

[8] Shirokov B. M. The distribution of the $d(n, \omega)$ in residue classes. Tr. Petrozavodsk. Gos. Univ. Ser. Mat., 1995, vol. 2, pp. 136-144 (in Russian).

[9] Shirokov B. M. The distribution of values of the extended sum of divisors. Tr. Petrozavodsk. Gos. Univ. Ser. Mat., 1996, vol. 3, pp. 176-189 (in Russian).

[10] Fomenko O. M. A distribution of the values of the multiplicative functions prime modulo. Zapiski nauch. sem. LOMI., 1980, vol. 93, pp. 218-224.

[11] Delange H. Sur la distribution des entiers ayant certaines proprietes. Ann. Sci. Ecole norm. super., 1956, vol. 73, no. 1, pp. 15-74.

[12] Trudgian T. The sum of the unitary divisor function. Publ. de l'Inst. Math. Nouvelle śerie., 2015, vol. 97(111), pp. 175-180.

[13] Prachar K. Prime number distribution. M.: Mir, 1967.

[14] Shirokov B. M. On the tauber type theorem. Materials of the international school-conference on the theory of functions of the complex variable. Petrozavodsk: Publ. PetrGU, 2004, pp. 14-15 (in Russian).

Received May 11, 2016.

In revised form, October 8, 2016.

Accepted October 8, 2016.

Petrozavodsk State University

33, Lenina st., 185910 Petrozavodsk, Russia

E-mail: bmshir@mail.ru,gromak_la@mail.ru 